www.nature.com/pj

\title{
Amphiphilic POSS-core dendrons for optically transparent thermoplastic films with tunable wettability
}

\author{
Koichi Ogi, Sakina Miyauchi and Kensuke Naka
}

Second- and third-generation hydroxy-terminated poly(amidoamine)-type heptaisobutyl-polyhedral oligomeric silsesquioxane (POSS)-core dendrons (that is, G2POSS-OH and G3POSS-OH, respectively) were prepared by ester-amide exchange reaction of G2 and G3 methyl acrylate-terminated POSS-core dendrons, which were synthesized from aminopropyl heptaisobutyl-T8silsesquioxane via multiple Michael and ester-amide exchange reactions in excess amounts of methyl acrylate and ethanolamine, respectively. Carboxylic acid-terminated second- and third-generation poly(amidoamine)-type heptaisobutyl-POSS-core dendrons (that is, G2POSS-COOH and G3POSS-COOH, respectively) were quantitatively obtained by treatment of second- and thirdgeneration $t$-butyl ester-terminated POSS-core dendrons with $\mathrm{HCO}_{2} \mathrm{H}$. The hydrodynamic sizes of G2POSS-OH and G3POSS-OH micelles in methanol decreased from $18 \pm 10$ to $6 \pm 4 \mathrm{~nm}$, respectively. Casting of a methanol solution of G3POSS-OH onto a glass substrate followed by heating at $100^{\circ} \mathrm{C}$ for $15 \mathrm{~min}$ provided optically transparent films. However, casting of a chloroform solution of G3POSS-OH resulted in the formation of an opaque whitish film. Both methanol and chloroform solutions of G2POSS-OH generated opaque whitish films. Casting of a $2.6 \mathrm{~m}$ formic acid solution of G3POSS-COOH and subsequent heating at $100^{\circ} \mathrm{C}$ for $15 \mathrm{~min}$ resulted in a highly transparent self-standing film. The peripheral functional groups may have been exposed on the surface of the cast films, which enabled control of the wettability of the films via the polarity of the $\mathrm{COOH}$ groups.

Polymer Journal (2015) 47, 259-266; doi:10.1038/pj.2014.119; published online 10 December 2014

\section{INTRODUCTION}

Polyhedral oligomeric silsesquioxanes (POSSs) have received increasing interest over the past decade, as important nano-building blocks primarily because of their well-defined structures, which include a $0.53 \mathrm{~nm}$ rigid inorganic core that can be linked to eight functional groups to produce organic-inorganic hybrid structures. ${ }^{1-3}$ Numerous strategies, both chemical and physical, have been explored to disperse POSSs into polymer matrices to achieve hybrid materials with interesting properties. POSS compounds have been used as fillers in polymer matrices to form organic-inorganic hybrid materials. ${ }^{4-7}$ Another method of using POSS compounds involves the direct cross-linking of POSS units with small organic molecules to form three-dimensional networks. ${ }^{8-10}$ POSS-containing polymers are particularly interesting as thermoplastic materials because they exhibit good thermal and mechanical properties and are highly resistant to oxidation. ${ }^{11-17}$ Controlled self-assembly of cubic silsesquioxane scaffolds in these polymers can enable precise tuning of their properties. An effective approach to controlling their nanometer-level structures is the addition of amphiphilic properties to the self-assembly of these developed well-defined hybrid materials. ${ }^{18-24}$ Among amphiphilic polymers, those with well-defined structures that contain hydrophobic POSS moieties have been synthesized, and the self-assembly behavior of these hybrid polymers has attracted significant attention. ${ }^{18-23}$ These polymers exhibit a phase-separated morphology in the bulk state. The hydrophobic POSS moieties self-assemble to form relatively large crystalline aggregates such as lamellar structures; ${ }^{25,26}$ however, precise control of the self-assembly structures of POSS units to achieve structures smaller than $10 \mathrm{~nm}$ is still difficult to achieve in a polymer matrix. Therefore, optically transparent materials based on these hybrid polymers are rarely obtained. In addition, the molecular-level surface chemical structures of films composed of POSS-containing amphiphilic polymers are difficult to control in these self-assembly systems.

Among the many different types of amphiphilic polymeric structures, amphiphilic dendrons have shown particularly interesting selfassembly behaviors and functional performances due to their unique cone-like shapes and multiple functionalities on the terminal groups. ${ }^{27,28}$ Their cone volumes can be precisely controlled by changing their generations. The combination of hydrophobic POSScores with hydrophilic dendrons could provide precise control of the self-assembly structures in solution, as well as the bulk state. The preparation of dendron-stabilized metal nanoparticles could offer enhanced stability and various interesting properties depending on the generation of the dendrons. ${ }^{29,30}$ We expect that the stabilities of the self-assembled structures of POSS-core dendrons are strongly dependent on the dendron generation and peripheral functional groups of the organic dendrons. In the present study, we design and synthesize heptaisobutyl-T8-silsesquioxy-core poly(amidoamine)-type 
dendrons with different generations and peripheral functional groups. The aim of this study is to obtain optically transparent films containing precisely controlled aggregate states. The introduction of dendrons to POSS structures may prevent the formation of lamellar self-assembly structures or larger aggregates because of their core-like structures. Therefore, they are expected to form optically transparent thermoplastic organic-inorganic hybrid films. The most significant advantage of the present system is that the peripheral functional groups would be located on the surface of the dendron assemblies because of the high proportion of terminal functional groups on the external surfaces of the dendrons. However, amphiphilic POSS dendrons, in which hydrophobic POSS cages are covalently bonded to hydrophilic dendron units, are seldom reported. To our knowledge, only one example, which is an urea/malonamide dendron-POSS nanohybrid, has been prepared. ${ }^{31}$ Coating these dendron-POSS compounds onto silicon wafer surfaces resulted in a porous morphology.

\section{EXPERIMENTAL PROCEDURE}

\section{Materials}

All of the solvents and chemicals used in this study were obtained as reagentgrade quality and used without further purification. All of the reactions were performed under a nitrogen atmosphere. Heptaisobutyl-aminopropyl-T8silsesquioxane (POSS- $\mathrm{NH}_{2}$ ) was obtained from Hybrid Plastics, Inc. (Hattiesburg, MS, USA).

\begin{abstract}
Measurements
${ }^{1} \mathrm{H}-(300$ or $400 \mathrm{MHz}),{ }^{13} \mathrm{C}-(75$ or $100 \mathrm{MHz})$ and ${ }^{29} \mathrm{Si}-(100 \mathrm{MHz})$ nuclear magnetic resonance (NMR) spectra were recorded on a BRUKER DPX-300 (Bruker Biospin GmbH, Rheinstetten, Germany). MALDI-TOF-MS was recorded on a BRUKER Autoflex II instrument (Bruker Daltonics, Billerica, MA, USA). High-resolution mass spectra were obtained on a JEOL JMS700 spectrometer (JEOL, Tokyo, Japan). Fourier transform infrared spectra were obtained on a JASCO FT/IR-4100 (JASCO, Tokyo, Japan) spectrometer using $\mathrm{KBr}$ pellets. UV-vis spectra were recorded on a JASCO spectrophotometer V-670 KKN (JASCO). Surface images were measured using tapping mode atomic force microscopy on a SHIMAZU SPM-9600 (SHIMAZU, Kyoto, Japan). A sample was prepared by depositing one drop of the desired solution on a silicon substrate, followed by drying at room temperature to remove the solvent. Dynamic light scattering measurements were conducted at room temperature using a Nicomp Model 370 instrument (NICOMP, Santa Barbara, CA, USA) with a $35 \mathrm{~mW}$ Ar-Ne laser as a light source. The POSS-core dendrons were dissolved in methanol at a concentration of $0.5 \mathrm{wt} \%$. Before the light scattering measurements, the sample solutions were passed through $0.45 \mu \mathrm{m}$ filters. A Nicomp model was used for data analysis to extract the average hydrodynamic size. Differential scanning calorimetry (DSC) was recorded on a TA Instruments 2920 Modulated DSC (TA Instruments, New Castle, DE, USA). Water contact angles were measured using a high-resolution digital camera (DS-Fi7, Nikon, Tokyo, Japan) at room temperature with water as the probe fluid $(1.5 \mathrm{ml})$. Each reported contact angle was an average value of at least five independent measurements.
\end{abstract}

\section{Synthesis}

G1POSS- $\mathrm{OCH}_{3}$. Methyl acrylate $(14.4 \mathrm{ml}, 160 \mathrm{mmol})$ was added via a syringe to a solution of POSS- $\mathrm{NH}_{2}(7.00 \mathrm{~g}, 8.02 \mathrm{mmol})$ in toluene $(30 \mathrm{ml})$ and methanol $(30 \mathrm{ml})$ under $\mathrm{N}_{2}$, and the resulting mixture was heated at $60^{\circ} \mathrm{C}$ for 2 days. After the volatiles were removed under reduced pressure, a white solid was obtained (yield: 98.8\%). ${ }^{1} \mathrm{H}-\mathrm{NMR}\left(\mathrm{CDCl}_{3}\right): \delta 0.58\left(\mathrm{~m}, 16 \mathrm{H}, \mathrm{Si}-\mathrm{CH}_{2}-\right.$ $\left.\mathrm{CH}_{2}-\mathrm{CH}_{2}-, \mathrm{Si}-\mathrm{CH}_{2}-\mathrm{CH}\left(\mathrm{CH}_{3}\right)_{2}\right), 0.94\left(\mathrm{~d}, 42 \mathrm{H}, \mathrm{Si}-\mathrm{CH}_{2}-\mathrm{CH}\left(\mathrm{CH}_{3}\right)_{2}, J=6.4-\right.$ $\mathrm{Hz}), 1.50\left(\mathrm{~m}, 2 \mathrm{H}, \mathrm{Si}-\mathrm{CH}_{2}-\mathrm{CH}_{2}-\mathrm{CH}_{2}-\right), 1.85\left(\mathrm{~m}, 7 \mathrm{H}, \mathrm{Si}-\mathrm{CH}_{2}-\mathrm{CH}\left(\mathrm{CH}_{3}\right)_{2}\right)$, $2.40\left(\mathrm{t}, 2 \mathrm{H}, \mathrm{Si}-\mathrm{CH}_{2}-\mathrm{CH}_{2}-\mathrm{CH}_{2}, J=7.6 \mathrm{~Hz}\right), 2.43\left(\mathrm{t}, 4 \mathrm{H},-\mathrm{CH}_{2}-\mathrm{CH}_{2}-\mathrm{CO}-\right.$, $J=7.4 \mathrm{~Hz}$ ), $2.76\left(\mathrm{t}, 4 \mathrm{H},-\mathrm{CH}_{2}-\mathrm{CH}_{2}-\mathrm{CO}-, J=7.2 \mathrm{~Hz}\right), 3.66(\mathrm{~s}, 6 \mathrm{H},-\mathrm{CO}-\mathrm{O}-$ $\left.\mathrm{CH}_{3}\right) .{ }^{13} \mathrm{C}-\mathrm{NMR}\left(\mathrm{CDCl}_{3}\right): \delta 9.59,20.26,22.47,23.70,25.67,32.56,49.19$, 51.47, 56.47, 173.03. ${ }^{29} \mathrm{Si}-\mathrm{NMR}\left(\mathrm{CDCl}_{3}\right): \delta-67.22,-67.71,-67.90$. FT-IR
(KBr): $\nu=431,483,743,833,1105,1230,1332,1366,1465,1743,2954,3448$ $\mathrm{cm}^{-1}$. HR FAB-MS: calcd for $\mathrm{C}_{39} \mathrm{H}_{84} \mathrm{NO}_{16} \mathrm{Si}_{8}\left[\mathrm{M}+\mathrm{H}^{+}\right] \mathrm{m} / z$ 1046.3946; found 1046.3942.

G1POSS- $\mathrm{NH}_{2}$. Ethylenediamine $(7.51 \mathrm{ml}, 112 \mathrm{mmol})$ was added to a solution of G1POSS- $\mathrm{OCH}_{3}(11.8 \mathrm{~g}, 11.3 \mathrm{mmol})$ in toluene $(25 \mathrm{ml})$ and methanol $(25 \mathrm{ml})$ under $\mathrm{N}_{2}$, and the resulting mixture was heated at $60^{\circ} \mathrm{C}$ for 3 days. After the volatiles were removed under reduced pressure, the residue was dissolved in toluene/hexane $(1 / 1, \mathrm{v} / \mathrm{v})$. The volatiles were again removed under reduced pressure. This process was repeated five times to obtain a white solid (yield: 99\%). ${ }^{1} \mathrm{H}-\mathrm{NMR}\left(\mathrm{CDCl}_{3}\right): \delta 0.59\left(\mathrm{~m}, 16 \mathrm{H}, \mathrm{Si}-\mathrm{CH}_{2}-\mathrm{CH}\left(\mathrm{CH}_{3}\right)_{2}, \mathrm{Si}-\mathrm{CH}_{2}-\right.$ $\left.\mathrm{CH}_{2}-\mathrm{CH}_{2}\right), 0.94\left(\mathrm{~d}, 42 \mathrm{H}, \mathrm{Si}-\mathrm{CH}_{2}-\mathrm{CH}\left(\mathrm{CH}_{3}\right)_{2}, J=6.7 \mathrm{~Hz}\right), 1.50(\mathrm{~m}, 2 \mathrm{H}$, $\left.\mathrm{Si}-\mathrm{CH}_{2}-\mathrm{CH}_{2}-\mathrm{CH}_{2}-\right), \quad 1.85\left(\mathrm{~m}, \quad 7 \mathrm{H}, \quad \mathrm{Si}-\mathrm{CH}_{2}-\mathrm{CH}\left(\mathrm{CH}_{3}\right)_{2}\right), 2.36(\mathrm{~m}, 4 \mathrm{H}$, $\left.-\mathrm{CH}_{2}-\mathrm{CH}_{2}-\mathrm{CO}-\right), 2.46\left(\mathrm{t}, 2 \mathrm{H}, \mathrm{Si}-\mathrm{CH}_{2}-\mathrm{CH}_{2}-\mathrm{CH}_{2}, J=7.8 \mathrm{~Hz}\right), 2.74(\mathrm{t}, 4 \mathrm{H}$ $\left.-\mathrm{CH}_{2}-\mathrm{CH}_{2}-\mathrm{NH}_{2}, J=6.7 \mathrm{~Hz}\right), 2.83\left(\mathrm{~m}, 4 \mathrm{H},-\mathrm{CH}_{2}-\mathrm{CH}_{2}-\mathrm{CO}-\right), 3.30$ (b, $\left.4 \mathrm{H},-\mathrm{CH}_{2}-\mathrm{CH}_{2}-\mathrm{NH}_{2}\right) .{ }^{13} \mathrm{C}-\mathrm{NMR}\left(\mathrm{CDCl}_{3}\right): \delta 9.68,19.08,22.48,23.85,25.70$, $33.93,35.75,45.35,49.67,56.25,172.82 .{ }^{29} \mathrm{Si}-\mathrm{NMR}\left(\mathrm{CDCl}_{3}\right): \delta-67.60$, $-67.71,-67.90$. FT-IR (KBr): $\nu=431,481,742,837,1106,1230,1332$, 1366, 1465, 1560, 1654, 2365, 2954, $3422 \mathrm{~cm}^{-1}$. HR FAB-MS: calcd for $\mathrm{C}_{41} \mathrm{H}_{92} \mathrm{~N}_{5} \mathrm{O}_{14} \mathrm{Si}_{8}\left[\mathrm{M}+\mathrm{H}^{+}\right] \mathrm{m} / z$ 1102.4797; found, 1102.4786 .

G2POSS-OCH - Methyl acrylate $(29.8 \mathrm{ml}, 331 \mathrm{mmol})$ was added via a syringe to a solution of G1POSS- $\mathrm{NH}_{2}(9.12 \mathrm{~g}, 8.27 \mathrm{mmol})$ in toluene $(20 \mathrm{ml})$ and methanol $(25 \mathrm{ml})$ under $\mathrm{N}_{2}$, and the resulting mixture was heated at $60^{\circ} \mathrm{C}$ for 3 days. After the volatiles were removed under reduced pressure, a white solid was obtained (yield: 97.1\%). ${ }^{1} \mathrm{H}-\mathrm{NMR}\left(\mathrm{CDCl}_{3}\right): \delta 0.59\left(\mathrm{~m}, 16 \mathrm{H}, \mathrm{Si}-\mathrm{CH}_{2}-\mathrm{CH}\right.$ $\left.\left(\mathrm{CH}_{3}\right)_{2}, \mathrm{Si}-\mathrm{CH}_{2}-\mathrm{CH}_{2}-\mathrm{CH}_{2}-\right), 0.94\left(\mathrm{~d}, 42 \mathrm{H}, \mathrm{Si}-\mathrm{CH}_{2}-\mathrm{CH}\left(\mathrm{CH}_{3}\right)_{2}, J=6.7 \mathrm{~Hz}\right)$, $1.53\left(\mathrm{~m}, 2 \mathrm{H}, \mathrm{Si}-\mathrm{CH}_{2}-\mathrm{CH}_{2}-\mathrm{CH}_{2}\right), 1.86\left(\mathrm{~m}, 7 \mathrm{H}, \mathrm{Si}-\mathrm{CH}_{2}-\mathrm{CH}\left(\mathrm{CH}_{3}\right)_{2}\right), 2.36(\mathrm{~m}$, $\left.4 \mathrm{H},-\mathrm{CH}_{2}-\mathrm{CH}_{2}-\mathrm{CO}-\mathrm{NH}-\right), 2.43\left(\mathrm{t}, 8 \mathrm{H},-\mathrm{CH}_{2}-\mathrm{CH}_{2}-\mathrm{CO}-\mathrm{OCH}_{3}, J=6.7 \mathrm{~Hz}\right)$, 2.47 (b, $2 \mathrm{H}, \mathrm{Si}-\mathrm{CH}_{2}-\mathrm{CH}_{2}-\mathrm{CH}_{2}$ ), 2.54 (t, $4 \mathrm{H},-\mathrm{NH}-\mathrm{CH}_{2}-\mathrm{CH}_{2}-\mathrm{N}-, J=5.9 \mathrm{~Hz}$ ), $2.76\left(\mathrm{t}, 8 \mathrm{H},-\mathrm{CH}_{2}-\mathrm{CH}_{2}-\mathrm{CO}-\mathrm{OCH}_{3}, J=6.8 \mathrm{~Hz}\right), 2.80\left(\mathrm{t}, 4 \mathrm{H},-\mathrm{CH}_{2}-\mathrm{CH}_{2}-\mathrm{CO}-\right.$ $\mathrm{NH}-, J=7.7 \mathrm{~Hz}$ ), $3.29\left(\mathrm{~m}, 4 \mathrm{H},-\mathrm{NH}-\mathrm{CH}_{2}-\mathrm{CH}_{2}-\mathrm{N}-\right), 3.67(\mathrm{~s}, 12 \mathrm{H},-\mathrm{CO}-\mathrm{O}-$ $\left.\mathrm{CH}_{3}\right) .{ }^{13} \mathrm{C}-\mathrm{NMR}\left(\mathrm{CDCl}_{3}\right): \delta 9.71,18.86,22.45,23.83,25.66,32.68,33.47,37.00$, $49.34,51.42,53.05,56.05,172.07,172.90 .{ }^{29} \mathrm{Si}-\mathrm{NMR}\left(\mathrm{CDCl}_{3}\right): \delta-67.48$ $-67.65,-67.78,-67.98$. FT-IR (KBr): $\nu=431,484,566,742,837,1105,1230$, $1332,1366,1465,1542,1654,1738,2954,3406 \mathrm{~cm}^{-1}$. HR FAB-MS: calcd for $\mathrm{C}_{57} \mathrm{H}_{116} \mathrm{~N}_{5} \mathrm{O}_{22} \mathrm{Si}_{8}\left[\mathrm{M}+\mathrm{H}^{+}\right] \mathrm{m} / z$ 1446.6268; found 1446.6272 .

G2POSS-OH. Ethanolamine $(1.00 \mathrm{ml}, 16.5 \mathrm{mmol})$ was added to a solution of G2POSS- $\mathrm{OCH}_{3}(1.32 \mathrm{~g}, 1.01 \mathrm{mmol})$ in toluene $(10 \mathrm{ml})$ and methanol $(20 \mathrm{ml})$ under $\mathrm{N}_{2}$, and the resulting mixture was heated at $60^{\circ} \mathrm{C}$ for 7 days. After the volatiles were removed under reduced pressure, the crude product was purified by preparative size-exclusion chromatography (LH-20) using methanol as the eluent to remove the unreacted products. The yield of pure G2POSS-OH was $36 \% .{ }^{1} \mathrm{H}-\mathrm{NMR}$ (methanol- $\left.\mathrm{d}_{4}\right): \delta 0.59\left(\mathrm{~m}, 16 \mathrm{H}, \mathrm{Si}-\mathrm{CH}_{2}-\mathrm{CH}\left(\mathrm{CH}_{3}\right)_{2}, \mathrm{Si}-\mathrm{CH}_{2}-\right.$ $\left.\mathrm{CH}_{2}-\mathrm{CH}_{2}-\right), 0.94\left(\mathrm{~d}, 42 \mathrm{H}, \mathrm{Si}-\mathrm{CH}_{2}-\mathrm{CH}\left(\mathrm{CH}_{3}\right)_{2}, J=6.4 \mathrm{~Hz}\right), 1.53(\mathrm{~b}, 2 \mathrm{H}, \mathrm{Si}-$ $\left.\mathrm{CH}_{2}-\mathrm{CH}_{2}-\mathrm{CH}_{2}-\right), 1.85$ (m, 7H, Si-CH $\left.\mathrm{CH}_{2}-\mathrm{CH}\left(\mathrm{CH}_{3}\right)_{2}\right), 2.38\left(\mathrm{~b}, 12 \mathrm{H},-\mathrm{CH}_{2}-\right.$ $\mathrm{CH}_{2}-\mathrm{CO}-$ ), 2.52 (b, 6H, Si- $\mathrm{CH}_{2}-\mathrm{CH}_{2}-\mathrm{CH}_{2},-\mathrm{NH}-\mathrm{CH}_{2}-\mathrm{CH}_{2}-\mathrm{N}-$ ), 2.72 (b, $12 \mathrm{H},-\mathrm{CH}_{2}-\mathrm{CH}_{2}-\mathrm{CO}-$ ), 3.25 (b, $4 \mathrm{H},-\mathrm{NH}-\mathrm{CH}_{2}-\mathrm{CH}_{2}-\mathrm{N}-$ ), 3.36 (b, $8 \mathrm{H},-\mathrm{NH}-$ $\left.\mathrm{CH}_{2}-\mathrm{CH}_{2}-\mathrm{OH}\right), 3.66$ (b, $\left.8 \mathrm{H},-\mathrm{NH}-\mathrm{CH}_{2}-\mathrm{CH}_{2}-\mathrm{OH}\right) .{ }^{13} \mathrm{C}-\mathrm{NMR}\left(\mathrm{CDCl}_{3}\right): \delta$ 9.91, 18.34, 22.46, 23.83, 25.70, 33.19, 33.49, 37.76, 42.06, 49.09, 50.60, 53.04, 56.01, 61.07, 172.98, 173.44. ${ }^{29} \mathrm{Si}-\mathrm{NMR}\left(\mathrm{CDCl}_{3}\right): \delta-67.71,-67.84,-67.88$. FT-IR $(\mathrm{KBr}): \nu=431,482,743,837,1104,1230,1332,1366,1465,1560$, $1646,2345,2954,3302,3855 \mathrm{~cm}^{-1}$. HR FAB-MS: calcd for $\mathrm{C}_{61} \mathrm{H}_{128} \mathrm{~N}_{9} \mathrm{O}_{22} \mathrm{Si}_{8}$ $\left[\mathrm{M}+\mathrm{H}^{+}\right] \mathrm{m} / \mathrm{z}$ 1562.7330; found 1562.7336 .

G2POSS- $\mathrm{NH}_{2}$. Ethylenediamine $(12.3 \mathrm{ml}, 184 \mathrm{mmol})$ was added to a solution of $\mathrm{G}_{2} \mathrm{POSS}-\mathrm{OCH}_{3}(9.52 \mathrm{~g}, 6.58 \mathrm{mmol})$ in toluene $(10 \mathrm{ml})$ and methanol $(24 \mathrm{ml})$ under $\mathrm{N}_{2}$, and the resulting mixture was heated at $60^{\circ} \mathrm{C}$ for 5 days. After the volatiles were removed under reduced pressure, the residue was dissolved in toluene/hexane $(1 / 1, \mathrm{v} / \mathrm{v})$. The volatiles were again removed under reduced pressure. This process was repeated five times to obtain a pale yellow solid (yield: $73.1 \%) .{ }^{1} \mathrm{H}-\mathrm{NMR}\left(\mathrm{CDCl}_{3}\right): \delta 0.59\left(\mathrm{~m}, 16 \mathrm{H}, \mathrm{Si}-\mathrm{CH}_{2}-\mathrm{CH}\left(\mathrm{CH}_{3}\right)_{2}\right.$, $\left.\mathrm{Si}-\mathrm{CH}_{2}-\mathrm{CH}_{2}-\mathrm{CH}_{2}-\right), 0.94\left(\mathrm{~d}, 42 \mathrm{H}, \mathrm{Si}-\mathrm{CH}_{2}-\mathrm{CH}\left(\mathrm{CH}_{3}\right)_{2}, \mathrm{~J}=6.6 \mathrm{~Hz}\right), 1.54(\mathrm{~m}$, $\left.2 \mathrm{H}, \mathrm{Si}-\mathrm{CH}_{2}-\mathrm{CH}_{2}-\mathrm{CH}_{2}-\right), 1.85\left(\mathrm{~m}, 7 \mathrm{H}, \mathrm{Si}-\mathrm{CH}_{2}-\mathrm{CH}\left(\mathrm{CH}_{3}\right)_{2}\right), 2.36(\mathrm{t}, 12 \mathrm{H}$ $\left.-\mathrm{CH}_{2}-\mathrm{CH}_{2}-\mathrm{CO}-, \mathrm{J}=6.1 \mathrm{~Hz}\right), 2.46\left(\mathrm{t}, 6 \mathrm{H}, \mathrm{Si}-\mathrm{CH}_{2}-\mathrm{CH}_{2}-\mathrm{CH}_{2},-\mathrm{NH}-\mathrm{CH}_{2}-\right.$ $\left.\mathrm{CH}_{2}-\mathrm{N}-, J=7.7 \mathrm{~Hz}\right), 2.74\left(\mathrm{t}, 12 \mathrm{H},-\mathrm{CH}_{2}-\mathrm{CH}_{2}-\mathrm{CO}-, J=6.1 \mathrm{~Hz}\right), 2.82(\mathrm{t}, 8 \mathrm{H}$, $\left.-\mathrm{CH}_{2}-\mathrm{CH}_{2}-\mathrm{NH}_{2}, J=5.8 \mathrm{~Hz}\right), 3.28\left(\mathrm{~m}, 12 \mathrm{H}, \mathrm{CO}-\mathrm{NH}-\mathrm{CH}_{2}-\mathrm{CH}_{2}-\right) .{ }^{13} \mathrm{C}-\mathrm{NMR}$ 
$\left(\mathrm{CDCl}_{3}\right): \delta 9.91,19.22,22.50,23.91,25.71,34.02,35.99,41.44,41.90,49.77$, 56.23, 172.60, 172.75. 29Si-NMR $\left(\mathrm{CDCl}_{3}\right): \delta-67.01,-67.77,-67.92 . \mathrm{FT}-\mathrm{IR}$ $(\mathrm{KBr}): \nu=432,484,742,837,1106,1230,1332,1366,1465,1560,1654,2345$, $2954,3423,3650,3752,3855 \mathrm{~cm}^{-1}$.

G3POSS $-\mathrm{OCH}_{3}$. Methyl acrylate $(19.3 \mathrm{ml}, 214 \mathrm{mmol})$ was added via a syringe to a solution of G2POSS- $\mathrm{NH}_{2}(4.17 \mathrm{~g}, 2.68 \mathrm{mmol})$ in toluene $(10 \mathrm{ml})$ and methanol $(10 \mathrm{ml})$ under $\mathrm{N}_{2}$, and the resulting mixture was heated at $60{ }^{\circ} \mathrm{C}$ for 5 days. After the volatiles were removed under reduced pressure, the crude product was purified by preparative size-exclusion chromatography ( $\mathrm{LH}-20$ ) using methanol as the eluent to remove the unreacted products. The yield of pure G3POSS-OCH 3 as a white solid was $54.0 \%$. ${ }^{1} \mathrm{H}-\mathrm{NMR}\left(\mathrm{CDCl}_{3}\right): \delta 0.59(\mathrm{~m}$, $\left.16 \mathrm{H}, \mathrm{Si}-\mathrm{CH}_{2}-\mathrm{CH}\left(\mathrm{CH}_{3}\right)_{2}, \mathrm{Si}-\mathrm{CH}_{2}-\mathrm{CH}_{2}-\mathrm{CH}_{2}-\right), 0.94$ (d, $42 \mathrm{H}, \mathrm{Si}-\mathrm{CH}_{2}-\mathrm{CH}$ $\left.\left(\mathrm{CH}_{3}\right)_{2}, J=6.4 \mathrm{~Hz}\right), 1.51\left(\mathrm{~m}, 2 \mathrm{H}, \mathrm{Si}-\mathrm{CH}_{2}-\mathrm{CH}_{2}-\mathrm{CH}_{2}-\right), 1.85\left(\mathrm{~m}, 7 \mathrm{H}, \mathrm{Si}-\mathrm{CH}_{2}-\right.$ $\left.\mathrm{CH}\left(\mathrm{CH}_{3}\right)_{2}\right), 2.37$ (b, 4H, Si- $\left.\mathrm{CH}_{2}-\mathrm{CH}_{2}-\mathrm{CH}_{2}-\mathrm{N}-\mathrm{CH}_{2}-\mathrm{CH}_{2}-\mathrm{CO}-\right), 2.45$ (b, $\left.24 \mathrm{H},-\mathrm{CH}_{2}-\mathrm{CH}_{2}-\mathrm{CO}-\right), 2.56\left(\mathrm{~m}, 14 \mathrm{H}, \mathrm{Si}-\mathrm{CH}_{2}-\mathrm{CH}_{2}-\mathrm{CH}_{2},-\mathrm{NH}-\mathrm{CH}_{2}-\mathrm{CH}_{2}-\right.$ $\mathrm{N}-), 2.77$ (m, 28H, $\left.-\mathrm{CH}_{2}-\mathrm{CH}_{2}-\mathrm{CO}-\right), 3.27\left(\mathrm{~m}, 12 \mathrm{H},-\mathrm{NH}-\mathrm{CH}_{2}-\mathrm{CH}_{2}-\mathrm{N}-\right)$, $3.67\left(\mathrm{~s}, 24 \mathrm{H},-\mathrm{CO}-\mathrm{O}-\mathrm{CH}_{3}\right) .{ }^{13} \mathrm{C}-\mathrm{NMR}\left(\mathrm{CDCl}_{3}\right): \delta 9.91,18.86,22.48,23.84$, 25.70, 32.71, 33.42, 33.85, 37.19, 37.53, 49.26, 49.91, 50.37, 51.64, 52.58, 52.96, 55.72, 172.38, 173.06. ${ }^{29} \mathrm{Si}-\mathrm{NMR}\left(\mathrm{CDCl}_{3}\right): \delta-67.67,-67.74,-67.94$. FT-IR $(\mathrm{KBr}): \nu=481,742,839,1111,1230,1365,1439,1543,1655,1737,2366,2954$, $3398 \mathrm{~cm}^{-1}$. HR FAB-MS: calcd for $\mathrm{C}_{93} \mathrm{H}_{180} \mathrm{~N}_{13} \mathrm{O}_{34} \mathrm{Si}_{8}\left[\mathrm{M}+\mathrm{H}^{+}\right] \mathrm{m} / z$ 2247.0912; found 2247.0854 .

G3POSS-OH. Ethanolamine $(1.94 \mathrm{ml}, 32.1 \mathrm{mmol})$ was added to a solution of G3POSS- $\mathrm{CH}_{3}(0.905 \mathrm{~g}, 0.40 \mathrm{mmol})$ in toluene $(2.5 \mathrm{ml})$ and methanol $(5.0 \mathrm{ml})$ under $\mathrm{N}_{2}$, and the resulting mixture was heated at $60^{\circ} \mathrm{C}$ for 12 days. After the volatiles were removed under reduced pressure, the crude product was purified by preparative size-exclusion chromatography (LH-20) using methanol as the eluent to remove the unreacted products. The yield of pure G3POSS-OH was $52 \%$. ${ }^{1} \mathrm{H}-\mathrm{NMR}\left(\right.$ methanol- $\left._{4}\right): \delta 0.60\left(\mathrm{~b}, 16 \mathrm{H}, \mathrm{Si}-\mathrm{CH}_{2}-\mathrm{CH}\left(\mathrm{CH}_{3}\right)_{2}, \mathrm{Si}-\mathrm{CH}_{2}-\right.$ $\mathrm{CH}_{2}-\mathrm{CH}_{2}-$ ), 0.99 (b, 42H, Si-CH $\left.-\mathrm{CH}\left(\mathrm{CH}_{3}\right)_{2}\right), 1.59$ (b, 2H, Si-CH $\mathrm{CH}_{2}-\mathrm{CH}_{2}-$ $\mathrm{CH}_{2}-$ ), 1.86 (b, 7H, Si- $\left.\mathrm{CH}_{2}-\mathrm{CH}\left(\mathrm{CH}_{3}\right)_{2}\right), 2.37$ (b, 28H, $\left.-\mathrm{CH}_{2}-\mathrm{CH}_{2}-\mathrm{CO}-\right)$, 2.58 (b, $\left.14 \mathrm{H}, \mathrm{Si}-\mathrm{CH}_{2}-\mathrm{CH}_{2}-\mathrm{CH}_{2},-\mathrm{NH}-\mathrm{CH}_{2}-\mathrm{CH}_{2}-\mathrm{N}-\right), 2.78$ (b, 28H, $-\mathrm{CH}_{2}-\mathrm{CH}_{2}-$ CO-), 3.31 (b, 28H, -NH- $\left.\mathrm{CH}_{2}-\mathrm{CH}_{2}-\mathrm{N}-,-\mathrm{NH}-\mathrm{CH}_{2}-\mathrm{CH}_{2}-\mathrm{OH}\right), 3.61$ (b, $16 \mathrm{H}$, $\left.-\mathrm{NH}-\mathrm{CH}_{2}-\mathrm{CH}_{2}-\mathrm{OH}\right) .{ }^{13} \mathrm{C}-\mathrm{NMR}\left(\right.$ methanol- $\left._{4}\right): \delta 23.53,25.19,26.20,34.75$, $38.69,43.02,51.24,53.56,61.65,175.25 .{ }^{29} \mathrm{Si}-\mathrm{NMR}\left(\right.$ methanol- $\left._{4}\right): \delta-67.12$, -67.53, -67.76. FT-IR (KBr): $\nu=484,742,837,1107,1230,1366,1465,1559$, 1647, 2953, $3301 \mathrm{~cm}^{-1}$. MALDI-TOF-MS: calcd for $\mathrm{C}_{101} \mathrm{H}_{205} \mathrm{~N}_{21} \mathrm{O}_{34} \mathrm{Si}_{8}$ $[\mathrm{M}]^{+} \mathrm{m} / \mathrm{z} 2480.311$; found 2480.454 .

G2POSS- $t B u$. $\quad t$-Butyl acrylate $(1.2 \mathrm{ml}, 8.54 \mathrm{mmol})$ was added via a syringe to a solution of G1POSS-NH $\mathrm{NH}_{2}(0.800 \mathrm{~g}, 0.74 \mathrm{mmol})$ in toluene $(5.0 \mathrm{ml})$ and methanol $(5.0 \mathrm{ml})$ under $\mathrm{N}_{2}$, and the resulting mixture was heated at $60{ }^{\circ} \mathrm{C}$ for 5 days. After the volatiles were removed under reduced pressure, a pale yellow solid was obtained (yield: $81 \%) .{ }^{1} \mathrm{H}-\mathrm{NMR}\left(\mathrm{CDCl}_{3}\right): \delta 0.59\left(\mathrm{~m}, 16 \mathrm{H}, \mathrm{Si}-\mathrm{CH}_{2}-\right.$ $\left.\mathrm{CH}\left(\mathrm{CH}_{3}\right)_{2}, \mathrm{Si}-\mathrm{CH}_{2}-\mathrm{CH}_{2}-\mathrm{CH}_{2}-\right), 0.95$ (d, 42H, Si-CH $-\mathrm{CH}\left(\mathrm{CH}_{3}\right)_{2}, J=6.8-$ $\mathrm{Hz}$ ), 1.44 (s 36H, -CO-OC- $\left.\left(\mathrm{CH}_{3}\right)_{3}\right), 1.53$ (b, 2H, Si-CH $-\mathrm{CH}_{2}-\mathrm{CH}_{2}-$ ); 1.85 $\left(\mathrm{m}, 7 \mathrm{H}, \mathrm{Si}-\mathrm{CH}_{2}-\mathrm{CH}\left(\mathrm{CH}_{3}\right)_{2}\right), 2.23\left(\mathrm{~b}, 4 \mathrm{H},-\mathrm{CH}_{2}-\mathrm{CH}_{2}-\mathrm{CO}-\mathrm{NH}-\right), 2.35$ (t, 8H, $\left.-\mathrm{CH}_{2}-\mathrm{CH}_{2}-\mathrm{CO}-\mathrm{OC}-\left(\mathrm{CH}_{3}\right)_{3}, \quad J=6.8 \mathrm{~Hz}\right), 2.45\left(\mathrm{t}, 2 \mathrm{H}, \mathrm{Si}-\mathrm{CH}_{2}-\mathrm{CH}_{2}-\mathrm{CH}_{2}\right.$, $J=7.4 \mathrm{~Hz}), 2.54\left(\mathrm{t}, 4 \mathrm{H},-\mathrm{NH}-\mathrm{CH}_{2}-\mathrm{CH}_{2}-\mathrm{N}-, J=6.0 \mathrm{~Hz}\right), 2.79(\mathrm{~m}, 12 \mathrm{H},-$ $\left.\mathrm{CH}_{2}-\mathrm{CH}_{2}-\mathrm{CO}-\right), 3.29\left(\mathrm{~m}, 4 \mathrm{H},-\mathrm{NH}-\mathrm{CH}_{2}-\mathrm{CH}_{2}-\right) .{ }^{13} \mathrm{C}-\mathrm{NMR}\left(\mathrm{CDCl}_{3}\right): \delta 9.80$, $18.85,22.80,23.88,25.68,28.06,33.69,33.89,37.28,49.27,49.91,52.76,55.67$, 80.38, 171.99, 173.95. ${ }^{29} \mathrm{Si}-\mathrm{NMR}\left(\mathrm{CDCl}_{3}\right): \delta-67.46,-67.60,-67.78,-67.97$. FT-IR $(\mathrm{KBr}): \nu=433,479,743,846,1112,1229,1367,1460,1655,1730,2955$, $3434 \mathrm{~cm}^{-1}$. HR FAB-MS: calcd for $\mathrm{C}_{69} \mathrm{H}_{140} \mathrm{~N}_{5} \mathrm{O}_{22} \mathrm{Si}_{8}\left[\mathrm{M}+\mathrm{H}^{+}\right] \mathrm{m} / \mathrm{z} 1614.8146$; found 1614.8142 .

G2POSS-COOH. G2POSS-tBu $(0.695 \mathrm{~g}, 0.43 \mathrm{mmol})$ was treated with $100 \mathrm{ml}$ of $\mathrm{HCO}_{2} \mathrm{H}$ for $24 \mathrm{~h}$ at $80^{\circ} \mathrm{C}$. Then, the $\mathrm{HCO}_{2} \mathrm{H}$ was completely removed by azeotropic distillation with water under reduced pressure to quantitatively afford G2POSS-COOH as a white powder, which was used without purification. ${ }^{1} \mathrm{H}-\mathrm{NMR}\left(\mathrm{CDCl}_{3}\right)$ : $\delta 0.61\left(\mathrm{~b}, 16 \mathrm{H}, \mathrm{Si}-\mathrm{CH}_{2}-\mathrm{CH}\left(\mathrm{CH}_{3}\right)_{2}, \mathrm{Si}-\mathrm{CH}_{2}-\mathrm{CH}_{2}-\right.$ $\left.\mathrm{CH}_{2}-\right), 0.95$ (b, 42H, Si-CH $\left.-\mathrm{CH}\left(\mathrm{CH}_{3}\right)_{2}\right), 1.85$ (b, 7H, Si-CH $\left.-\mathrm{CH}\left(\mathrm{CH}_{3}\right)_{2}\right)$, 2.60 (b, $\left.12 \mathrm{H},-\mathrm{CH}_{2}-\mathrm{CH}_{2}-\mathrm{CO}-\right), 2.76\left(\mathrm{~b}, 6 \mathrm{H}, \mathrm{Si}-\mathrm{CH}_{2}-\mathrm{CH}_{2}-\mathrm{CH}_{2},-\mathrm{NH}-\mathrm{CH}_{2}-\right.$ $\mathrm{CH}_{2}-\mathrm{N}-$ ), 2.95 (b, 4H, $\left.-\mathrm{CH}_{2}-\mathrm{CH}_{2}-\mathrm{CO}-\mathrm{NH}-\right), 3.09$ (b, 8H, $-\mathrm{CH}_{2}-\mathrm{CH}_{2}-$ $\mathrm{COOH}), 3.39$ (b, $4 \mathrm{H},-\mathrm{NH}-\mathrm{CH}_{2}-\mathrm{CH}_{2}-\mathrm{N}-$ ). FT-IR (KBr): $\nu=484,742,838$, $1108,1230,1401,1466,1594,1655,2363,2954,3423,3855 \mathrm{~cm}^{-1}$.
G3POSS- $t$ Bu. $t$-Butyl acrylate $(7.44 \mathrm{ml}, 51.3 \mathrm{mmol})$ was added via a syringe to a solution of G2POSS-NH $\mathrm{NH}_{2}(2.015 \mathrm{~g}, 1.30 \mathrm{mmol})$ in toluene $(15 \mathrm{ml})$ and methanol $(15 \mathrm{ml})$ under $\mathrm{N}_{2}$, and the resulting mixture was heated at $60{ }^{\circ} \mathrm{C}$ for 6 days. After the volatiles were removed under reduced pressure, a pale yellow solid was obtained (yield: $57 \%) .{ }^{1} \mathrm{H}-\mathrm{NMR}\left(\mathrm{CDCl}_{3}\right): \delta 0.59\left(\mathrm{~m}, 16 \mathrm{H}, \mathrm{Si}-\mathrm{CH}_{2}-\right.$ $\left.\mathrm{CH}\left(\mathrm{CH}_{3}\right)_{2}, \mathrm{Si}-\mathrm{CH}_{2}-\mathrm{CH}_{2}-\mathrm{CH}_{2}-\right), 0.95$ (d, 42H, Si-CH $\left.-\mathrm{CH}\left(\mathrm{CH}_{3}\right)_{2}, J=6.8\right)$, $1.44\left(\mathrm{~s} 72 \mathrm{H},-\mathrm{CO}-\mathrm{C}-\left(\mathrm{CH}_{3}\right)_{3}\right), 1.50\left(\mathrm{~m}, 2 \mathrm{H}, \mathrm{Si}-\mathrm{CH}_{2}-\mathrm{CH}_{2}-\mathrm{CH}_{2}-\right), 1.85$ (m, 7H, $\left.\mathrm{Si}-\mathrm{CH}_{2}-\mathrm{CH}\left(\mathrm{CH}_{3}\right)_{2}\right), 2.23$ (b, 4H, Si- $\left.\mathrm{CH}_{2}-\mathrm{CH}_{2}-\mathrm{CH}_{2}-\mathrm{N}-\mathrm{CH}_{2}-\mathrm{CH}_{2}-\mathrm{CO}-\right)$, $2.35\left(\mathrm{~m}, 24 \mathrm{H},-\mathrm{CH}_{2}-\mathrm{CH}_{2}-\mathrm{CO}-\right), 2.54\left(\mathrm{~m}, 14 \mathrm{H}, \mathrm{Si}-\mathrm{CH}_{2}-\mathrm{CH}_{2}-\mathrm{CH}_{2},-\mathrm{NH}-\right.$ $\left.\mathrm{CH}_{2}-\mathrm{CH}_{2}-\mathrm{N}-\right), 2.73\left(\mathrm{~m}, 28 \mathrm{H},-\mathrm{CH}_{2}-\mathrm{CH}_{2}-\mathrm{O}-\right), 3.28\left(\mathrm{~m}, 12 \mathrm{H},-\mathrm{NH}-\mathrm{CH}_{2}-\right.$ $\left.\mathrm{CH}_{2}-\mathrm{N}-\right) .{ }^{13} \mathrm{C}-\mathrm{NMR}\left(\mathrm{CDCl}_{3}\right): \delta 9.94,18.49,22.52,23.87,25.71,28.15,33.53$, 33.76, 33.85, 37.41, 37.57, 49.32, 49.95, 52.72, 55.68, 80.46, 172.05, 172.24. ${ }^{29} \mathrm{Si}-\mathrm{NMR}\left(\mathrm{CDCl}_{3}\right): \delta-67.59,-67.73,-67.93$. FT-IR $(\mathrm{KBr}): \nu=482,742,847$, 1120, 1229, 1367, 1460, 1543, 1655, 1729, 2955, $3321 \mathrm{~cm}^{-1}$. MALDI-TOF-MS: calcd for $\mathrm{C}_{117} \mathrm{~N}_{13} \mathrm{O}_{34} \mathrm{H}_{227} \mathrm{Si}_{8}[\mathrm{M}]^{+} m / z$ 2583.468; found 2585.652 .

G3POSS-COOH. G3POSS-tBu $(2.57 \mathrm{~g}, 0.610 \mathrm{mmol})$ was treated with $50 \mathrm{ml}$ of $\mathrm{HCO}_{2} \mathrm{H}$ for 2 days at $80^{\circ} \mathrm{C}$. Then, the $\mathrm{HCO}_{2} \mathrm{H}$ was completely removed by azeotropic distillation with water under reduced pressure to quantitatively afford G3POSS-COOH as a white powder, which was used without purification. ${ }^{1} \mathrm{H}-\mathrm{NMR}\left(\mathrm{CDCl}_{3}\right): \delta 0.60\left(\mathrm{~m}, 16 \mathrm{H}, \mathrm{Si}-\mathrm{CH}_{2}-\mathrm{CH}\left(\mathrm{CH}_{3}\right)_{2}, \mathrm{Si}-\mathrm{CH}_{2}-\mathrm{CH}_{2}-\right.$ $\left.\mathrm{CH}_{2}-\right), 0.95\left(\mathrm{~d}, 42 \mathrm{H}, \mathrm{Si}-\mathrm{CH}_{2}-\mathrm{CH}\left(\mathrm{CH}_{3}\right)_{2}, J=6.4 \mathrm{~Hz}\right), 1.53\left(\mathrm{~b}, 2 \mathrm{H}, \mathrm{Si}-\mathrm{CH}_{2}-\right.$ $\left.\mathrm{CH}_{2}-\mathrm{CH}_{2}-\right), 1.85$ (b, 7H, Si- $\left.\mathrm{CH}_{2}-\mathrm{CH}\left(\mathrm{CH}_{3}\right)_{2}\right), 2.5 \sim 3.8$ (b, 82H, Si- $\mathrm{CH}_{2}-$ $\left.\mathrm{CH}_{2}-\mathrm{CH}_{2},-\mathrm{CH}_{2}-\mathrm{CH}_{2}-\mathrm{CO}-,-\mathrm{NH}-\mathrm{CH}_{2}-\mathrm{CH}_{2}-\mathrm{N}-\right)$. FT-IR (KBr): $\nu=481,741$, $838,1108,1230,1400,1466,1591,1656,2954,3423,3855 \mathrm{~cm}^{-1}$.

\section{RESULTS AND DISCUSSION}

\section{Synthesis and characterization of POSS-core dendrons}

Hydroxy- and carboxylic acid-terminated POSS-core dendrons were synthesized from aminopropyl heptaisobutyl-T8-silsesquioxane (POSS- $\mathrm{NH}_{2}$ ) via consecutive Michael and ester-amide exchange reactions (Scheme 1). Although a methanol solution was generally used for both reactions, POSS- $\mathrm{NH}_{2}$ was insoluble in polar solvents and soluble in toluene and chloroform. When neat toluene was used as the solvent for the Michael reaction of POSS- $\mathrm{NH}_{2}$ with 5.0 equivalents of methyl acrylate, the reaction conversion was only $50 \%$, as estimated by ${ }^{1} \mathrm{H}-\mathrm{NMR}$ spectroscopy, even after heating at $60{ }^{\circ} \mathrm{C}$ for 2 days. No increase in the conversion was observed even after 7 days. When a mixed solvent system (that is, methanol/toluene, $1 / 1 \mathrm{v} / \mathrm{v}$ ) was used, quantitative conversion of POSS- $\mathrm{NH}_{2}$ with methyl acrylate was observed after heating at $60^{\circ} \mathrm{C}$ for 2 days. Therefore, the mixed solvent system (methanol/toluene, $1 / 1 \mathrm{v} / \mathrm{v}$ ) was used for all of the following reactions. Second- and third-generation hydroxy-terminated poly(amidoamine)-type heptaisobutyl-POSS dendrons (that is, G2POSS-OH and G3POSS-OH, respectively) were prepared by esteramide exchange of G2 and G3 methyl acrylate-terminated POSS-core dendrons (that is, G2POSS- $\mathrm{OCH}_{3}$ and G3POSS-OCH 3 , respectively) using excess ethanolamine. The products were purified using preparative size-exclusion chromatography to remove any unreacted compounds.

The structures of G2POSS-OH and G3POSS-OH were identified using multinuclear NMR and mass spectroscopy (Supplementary Information). The FAB-MS results provided conclusive evidence of the formation of G2POSS-OH. The MALDI-TOF-MS spectrum of G3POSS-OH also agreed with the theoretical value. The degree of functionalization of the dendrons with methoxy groups in G3POSS$\mathrm{OCH}_{3}$ was $94 \%$, as estimated using ${ }^{1} \mathrm{H}-\mathrm{NMR}$ spectroscopy by calculating and comparing the areas of the peaks corresponding to the methyl ester protons with those of the methylene protons on the POSS core.

Second- and third-generation $t$-butyl ester-terminated POSS-core dendrons (that is, G2POSS-tBu and G3POSS-tBu, respectively) were prepared as intermediates to the carboxylic acid-terminated POSS-core 


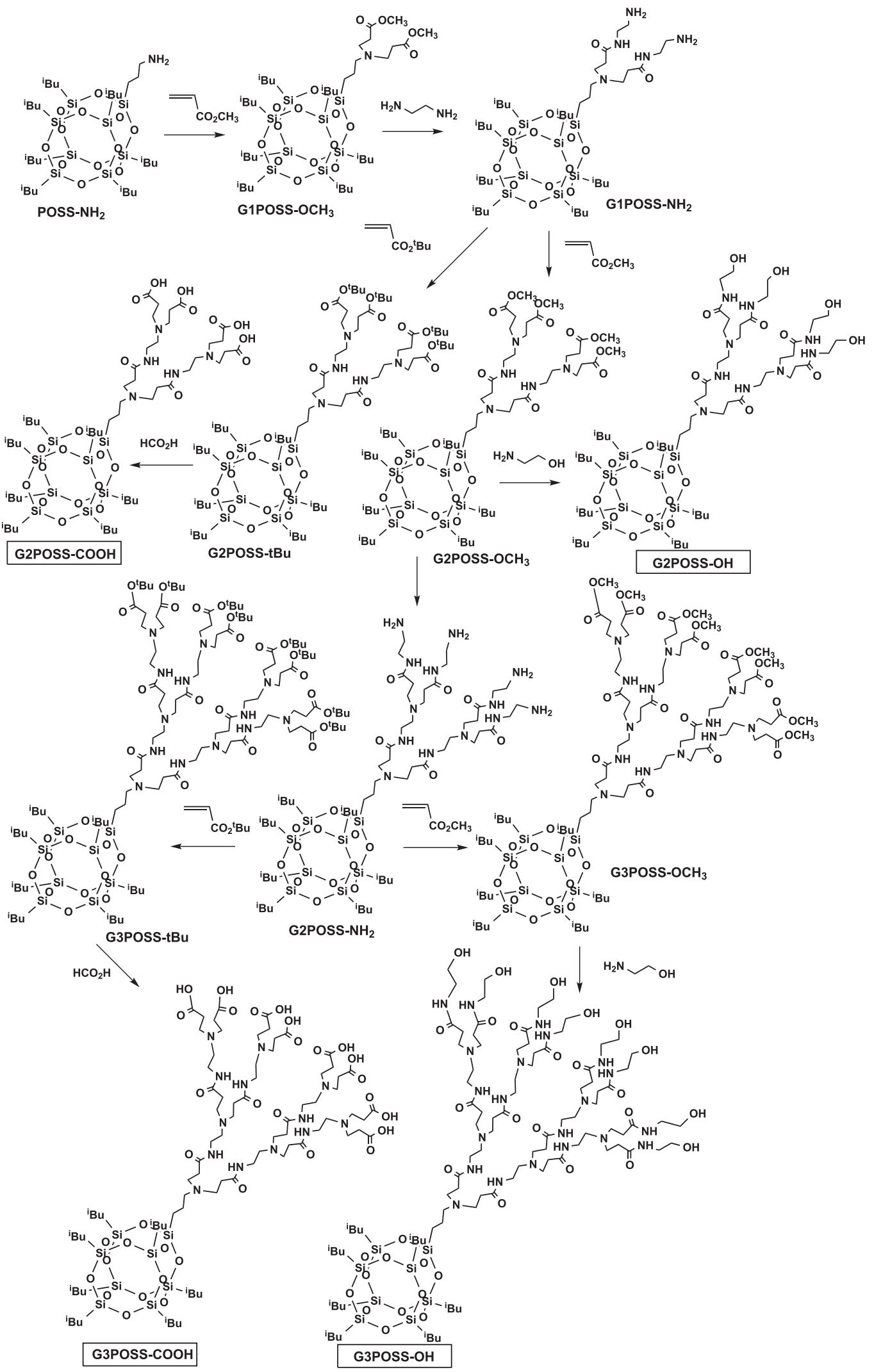

Scheme 1 Synthesis of POSS-core dendrons. 
dendrons. ${ }^{32}$ The structures of G2POSS-tBu and G3POSS-tBu were identified using multinuclear NMR and mass spectroscopy (Supplementary Information). The degree of functionalization of the dendrons with $t$-butyl groups in G3POSS-tBu was $97 \%$, as estimated using ${ }^{1} \mathrm{H}-\mathrm{NMR}$ spectroscopy. The MALDI-TOF-MS spectrum of G3POSS-tBu agreed with the theoretical value. Second- and thirdgeneration carboxylic acid-terminated POSS-core dendrons (that is, G2POSS-COOH and G3POSS-COOH, respectively) were quantitatively obtained by treatment of the $t$-butyl ester-terminated POSS-core dendrons with $\mathrm{HCO}_{2} \mathrm{H}$. After heating the mixtures at $80^{\circ} \mathrm{C}$ for 1 day, excess $\mathrm{HCO}_{2} \mathrm{H}$ was removed by azeotropic distillation with water under reduced pressure. Complete deprotection and lack of decomposition of the POSS core were confirmed by ${ }^{1} \mathrm{H}-\mathrm{NMR}$ spectroscopy and FT-IR analyses.

\section{Solution behaviors}

Although POSS- $\mathrm{NH}_{2}$ and G1POSS- $\mathrm{NH}_{2}$ were soluble in toluene and chloroform and insoluble in ethanol and methanol, G2POSS-OH was soluble in both ethanol and methanol, whereas G3POSS-OH was less soluble in hydrophobic solvents such as toluene and chloroform. G2POSS-COOH was soluble in chloroform and insoluble in both hydrophobic solvents, such as hexane and toluene and hydrophilic

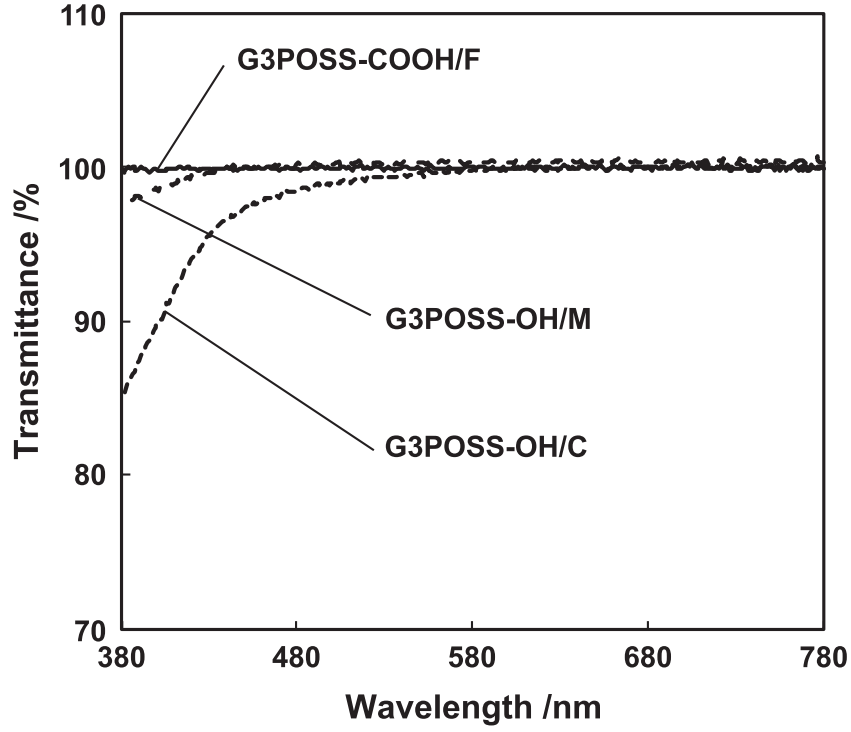

Figure 2 UV-vis spectra of G3POSS-OH/M, G3POSS-OH/C and G3POSS$\mathrm{COOH} / \mathrm{F}$ on glass substrates. The film thicknesses were approximately 0.6 , 0.8 and $0.7 \mu \mathrm{m}$, respectively.

\section{a}

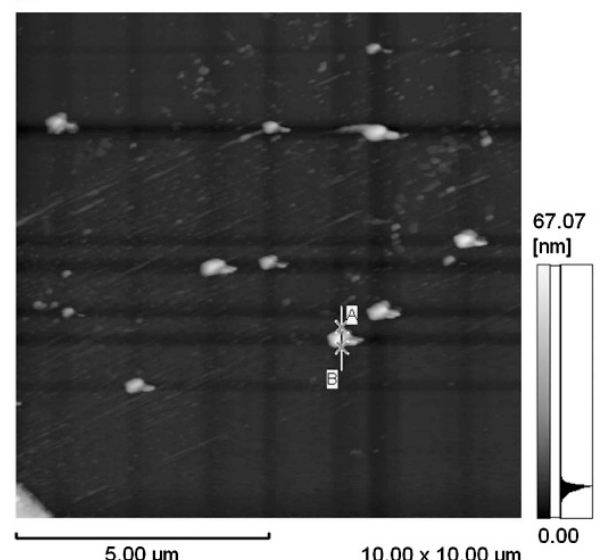

b

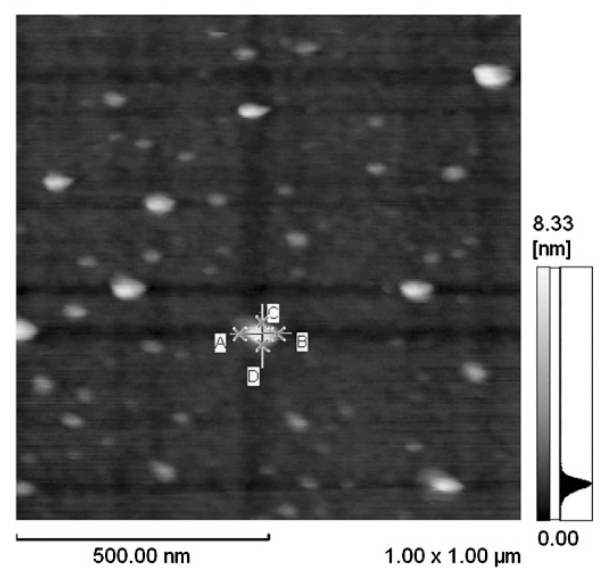

A-B

59.80

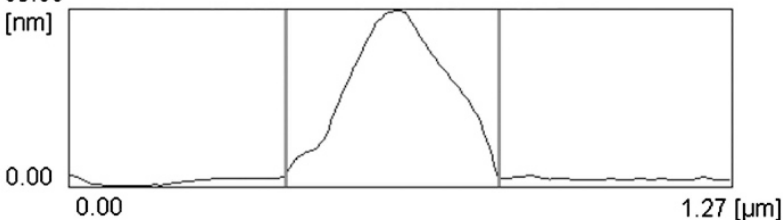

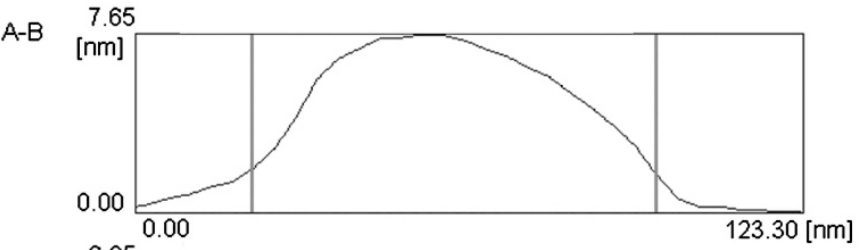

$\underline{\text { C-D }}$

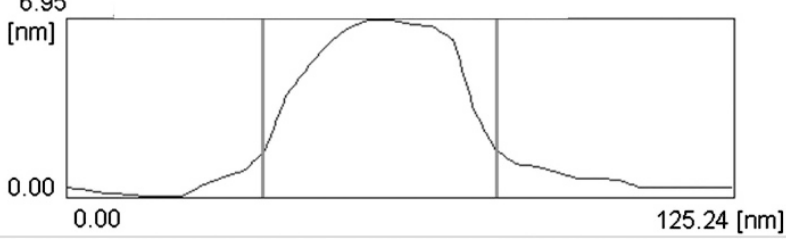

Figure 1 TM-AFM images of micelles of (a) G2POSS-OH and (b) G3POSS-OH. The samples were prepared under the conditions described in the Experimental procedure. A full color version of this figure is available at the Polymer Journal online. 
solvents such as ethanol and methanol. G3POSS-COOH was partially soluble in chloroform and fully soluble in only $\mathrm{HCO}_{2} \mathrm{H}$.

The hydrodynamic sizes of the micelles formed by the POSS-core dendrons (that is, G2POSS-OH and G3POSS-OH) in methanol were measured using dynamic light scattering at dendron concentrations of $0.5 \mathrm{wt} \%$. The micelle sizes of G2POSS-OH and G3POSS-OH decreased from $18 \pm 10$ to $6 \pm 4 \mathrm{~nm}$, respectively, thus increasing the generation numbers from two to three with the decreases of the micelle diameters. Tyndall's phenomenon was observed in a $2.6 \mathrm{M}$ formic acid solution of G3POSS-COOH $\left(1 \mathrm{mg} \mathrm{ml}^{-1}\right)$ upon irradiation with a laser pointer, which indicated that colloidal aggregates formed in this solution. The hydrodynamic size of the micelles formed by G3POSS-COOH in a $2.6 \mathrm{~m}$ formic acid solution using dynamic light scattering at dendron concentrations of $0.5 \mathrm{wt} \%$ was found to be $12 \pm 1 \mathrm{~nm}$. Because the octa isobutyl-substituted POSS has an approximate spherical diameter of $1.5 \mathrm{~nm}$, the hydrodynamic sizes of the micelles are reasonably explained as simple core-shell aggregate structures.

From tapping mode-AFM images of the dendrons on silicon substrates, the heights of the G2POSS-OH and G3POSS-OH micelles were determined to be 60 and $7 \mathrm{~nm}$, respectively (Figure 1). Although G2POSS-OH formed larger aggregates in the dry state, the G3POSS$\mathrm{OH}$ micelles retained their spherical form even on substrates. We assume that the micelle structure of G3POSS-OH was more stable because of the suitable characteristics of the core structures.

\section{Cast films}

Methanol and chloroform solutions of G3POSS-OH $\left(1 \mathrm{mg} \mathrm{ml}^{-1}\right)$ were casted onto glass substrates and heated at $100^{\circ} \mathrm{C}$ for $15 \mathrm{~min}$ using a hotplate. The methanol solution of G3POSS-OH formed a transparent film (that is, G3POSS-OH/M) with an optical transmittance greater than $98 \%$ in the visible region of $380-780 \mathrm{~nm}$ (Figure 2).
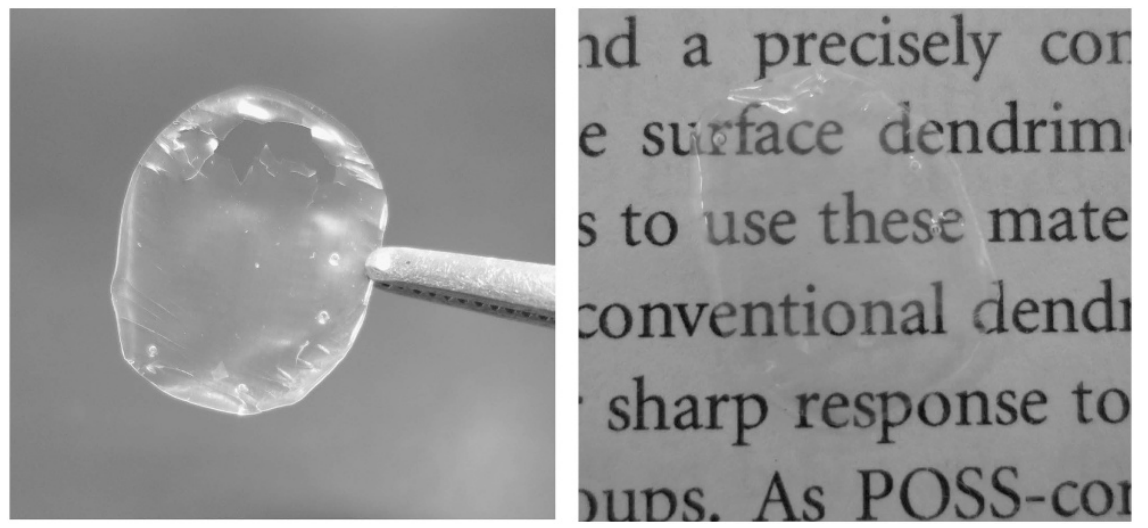

Figure 3 Photograph of a free-standing film obtained by casting a $2.6 \mathrm{~m}$ formic acid solution of G3POSS-COOH ( $10 \mathrm{mg} \mathrm{ml}^{-1}$ ) on a Teflon sheet and heating at $100{ }^{\circ} \mathrm{C}$ for $15 \mathrm{~min}$. A full color version of this figure is available at the Polymer Journal online.

a

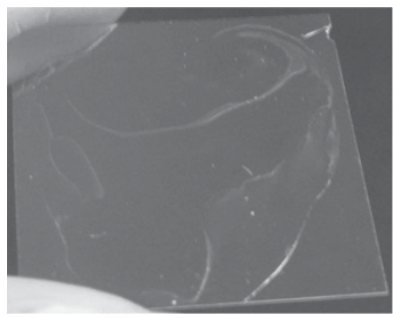

d

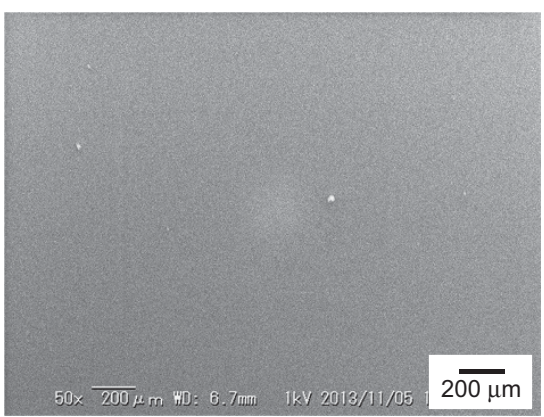

b

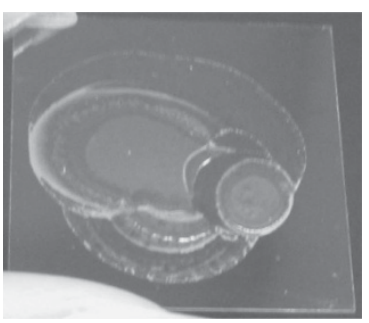

e

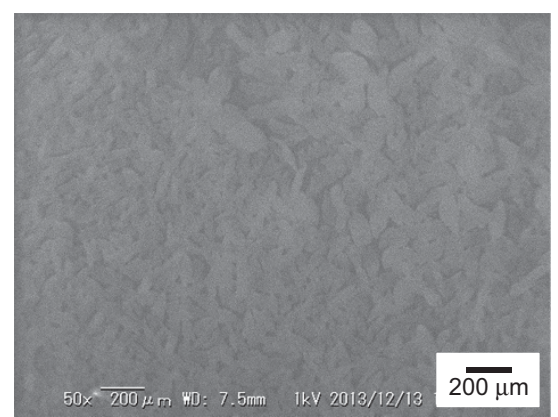

C

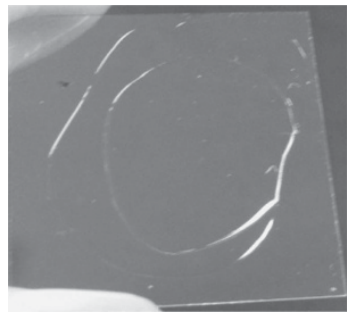

$\mathbf{f}$

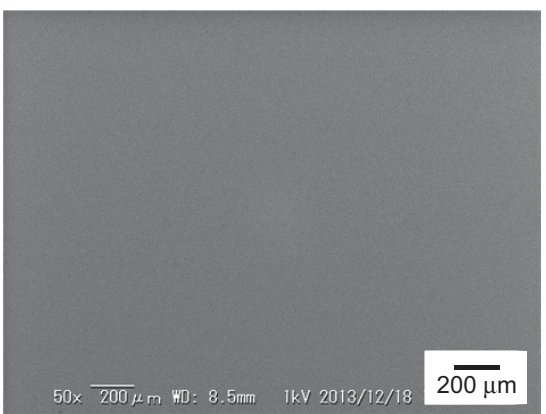

Figure 4 Appearances and s.e.m. images of (a and d) G3POSS-OH/M, (b and e) G3POSS-OH/C and (c and f) G3POSS-COOH/F on glass substrates. A full color version of this figure is available at the Polymer Journal online. 
In contrast, an opaque, whitish film (that is, G3POSS-OH/C) formed from the chloroform solution of G3POSS-OH. Both methanol and chloroform solutions of G2POSS-OH formed opaque, whitish films; the transmittances of these films at $600 \mathrm{~nm}$ were $80 \%$ and $56 \%$, respectively. The optical transmittance of the films generated from the methanol solution of G2POSS-OH was higher than that from the chloroform solution of G2POSS-OH.

The $2.6 \mathrm{M}$ formic acid solution of G3POSS-COOH $\left(1 \mathrm{mg} \mathrm{ml}^{-1}\right)$ was cast onto a glass substrate and heated at $100^{\circ} \mathrm{C}$ for $15 \mathrm{~min}$ using a hotplate. The optical transmittance of the resultant film (that is, G3POSS-COOH/F) was greater than $98 \%$ in the visible region of 380 $780 \mathrm{~nm}$ (Figure 2). Casting of a $2.6 \mathrm{~m}$ formic acid solution of G3POSS$\mathrm{COOH}\left(10 \mathrm{mg} \mathrm{ml}^{-1}\right)$ on a Teflon sheet and subsequent heating at $100^{\circ} \mathrm{C}$ for $15 \mathrm{~min}$ resulted in a free-standing film with high transparency (Figure 3).

The homogeneity of the cast films was confirmed using s.e.m.; the s.e. m. images of G3POSS-OH/M and G3POSS-COOH/F revealed smooth and homogeneous surfaces (Figure 4). However, the opaque whitish film generated from the chloroform solution of G3POSS-OH had a rough surface, which was attributed to the higher crystallinity of G3POSS-OH that induced a rough surface and increased the opacity of the film.

X-ray diffraction (XRD) analyses of powder samples of G3POSS-OH/ $\mathrm{M}$ and G3POSS-COOH/F showed intense peaks at $8.1^{\circ}$, which corresponded to microdomains of heptaisobutyl-POSS units (Figure 5). Diffraction peaks of the mono-functional alkyl cornerattached heptaisobutyl-POSS appeared at $2 \theta=8.2,11.0,12.2$ and $19.1^{\circ}$, and were associated with the hexagonal crystalline structure of POSS. $^{26,33}$ Although the XRD charts were dominated by broad hollow peaks at $\sim 20^{\circ}$, small peaks were also evident at $\sim 12^{\circ}$ and $19^{\circ}$. These data suggest that crystalline POSS units formed in the transparent cast films of both samples. The apparent crystallite sizes (L) of G3POSS-OH/ $\mathrm{M}$, G3POSS-OH/C and G3POSS-COOH/F were estimated to be $6.8,7.0$ and $5.1 \mathrm{~nm}$, respectively, based on the half-widths of the reflections at

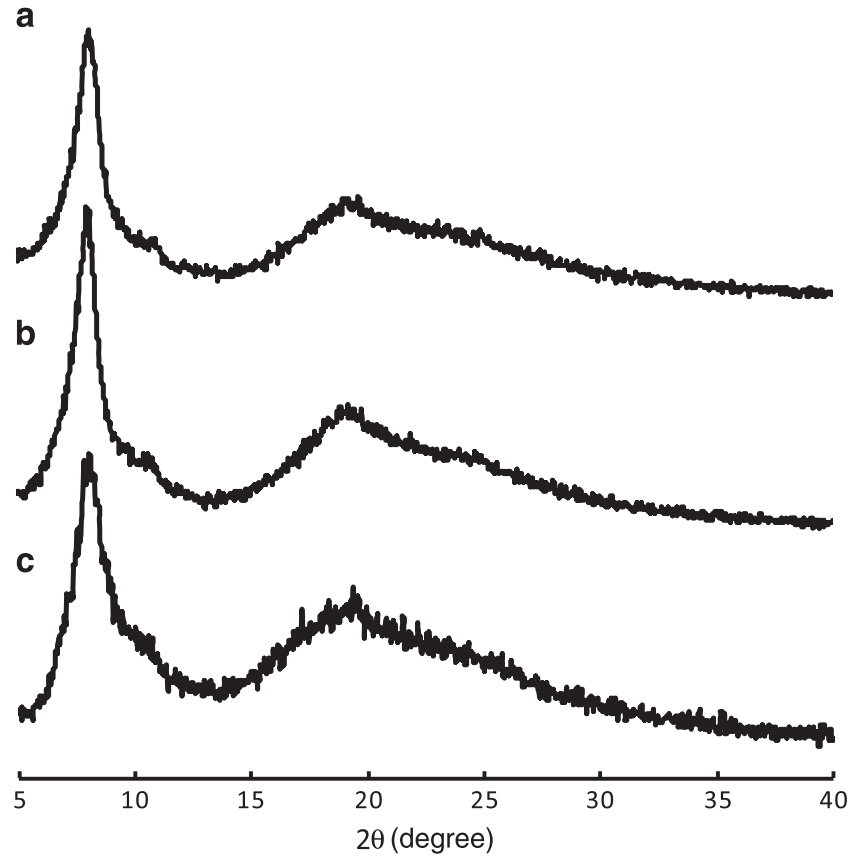

Figure 5 XRD patterns of (a) G3POSS-OH/M, (b) G3POSS-OH/C and (c) G3POSS- $\mathrm{COOH} / \mathrm{F}$. $8.2^{\circ}$ using Scherrer's equation $(L=\lambda / \beta \cos \theta$, where $\lambda$ is the wavelength of the X-ray, $\beta$ is the peak width at half maximum, and $\theta$ is the angle). These results indicated that we obtained optically transparent films containing precisely controlled aggregate states of the POSS units that were smaller than $10 \mathrm{~nm}$ via control of the number of generations.

The DSC curve of G3POSS-OH/M showed a glass-transition temperature at $9.6^{\circ} \mathrm{C}$ (Figure 6). Above the glass-transition temperature, the film became a paste form. In contrast, the DSC analysis of $\mathrm{G} 3 \mathrm{POSS}-\mathrm{COOH} / \mathrm{F}$ revealed a glass-transition temperature at $14.9^{\circ} \mathrm{C}$, and no softening behavior was observed above this temperature. This result may be due to effective intermolecular hydrogen bonding of the terminal $\mathrm{COOH}$ units.

It is expected that the peripheral functional groups may be exposed on the surface of the films, which would enable control of the wettability via the polarity of the $\mathrm{COOH}$ groups. The static contact angle for as-prepared G3POSS-COOH/F was determined to be $74 \pm 6^{\circ}$ (Figure 7); this angle significantly decreased to $<20^{\circ}$ after the film was immersed in a $0.025 \mathrm{M} \mathrm{NaOH}$ aqueous solution. Further treatment with acidic water $(0.08 \mathrm{~m}$ formic acid aqueous solution) increased the contact angle to $55 \pm 6^{\circ}$. However, treatment with distilled water

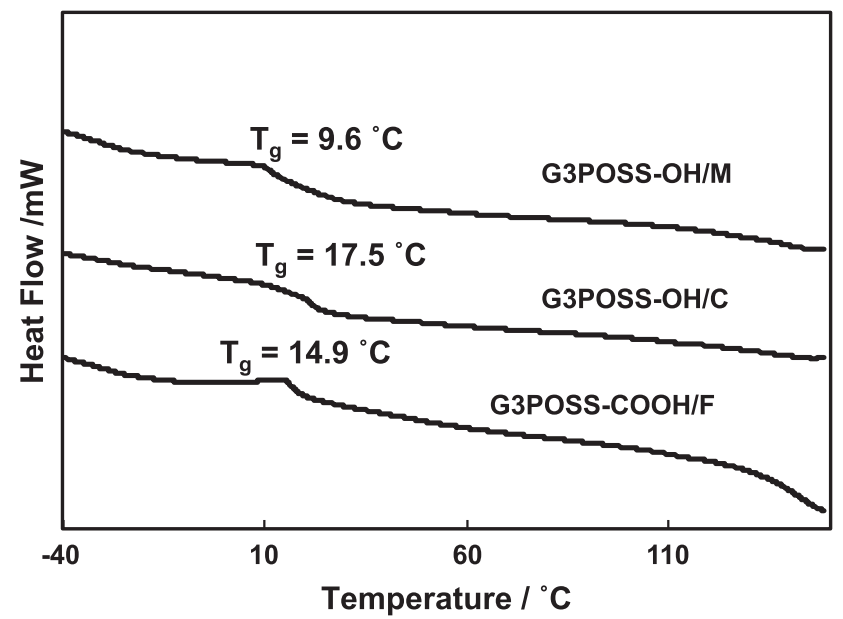

Figure 6 DSC traces of G3POSS-OH/M, G3POSS-OH/C and G3POSS-COOH/ $\mathrm{F}$ obtained at a heating rate of $10^{\circ} \mathrm{C} \mathrm{min}^{-1}$ under $\mathrm{N}_{2}$ flow over the entire temperature range.

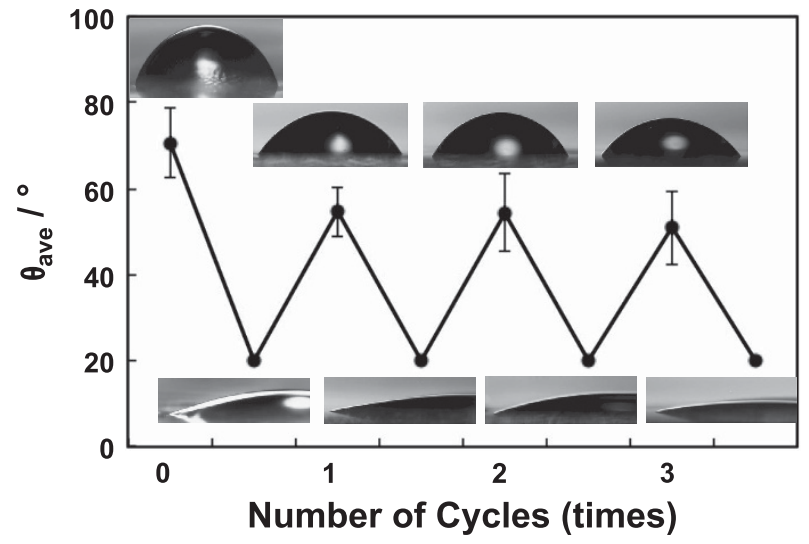

Figure 7 Reversible changes of the contact angles of $1.5 \mu \mathrm{l}$ water droplets on as-prepared G3POSS-COOH/F consecutively treated with a $0.025 \mathrm{~m} \mathrm{NaOH}$ aqueous solution and a $0.08 \mathrm{~m}$ formic acid aqueous solution. The insets show photographs of the water droplets on each surface. 
caused no change of the contact angle. These results suggest that the peripheral functional groups were exposed to the surface of the cast films because of the high proportion of terminal functional groups located at the external surfaces of the dendrons. Reproducible wettability changes of G3POSS- $\mathrm{COOH} / \mathrm{F}$ were achieved via consecutive treatment with a $0.025 \mathrm{M} \mathrm{NaOH}$ aqueous solution and acidic water ( $0.08 \mathrm{~m}$ formic acid aqueous solution). Several cycles were performed, and no substantial change of the contact angles was observed for at least four cycles (Figure 7).

\section{CONCLUSIONS}

Second- and third-generation hydroxy-terminated poly(amidoamine)type heptaisobutyl-POSS dendrons (that is, G2POSS-OH and G3POSS$\mathrm{OH}$, respectively) were prepared by ester-amide exchange of G2 and G3 methyl acrylate-terminated POSS-core dendrons, which were synthesized from aminopropyl heptaisobutyl-T8-silsesquioxane via multiple Michael and ester-amide exchange reactions with excess amounts of methyl acrylate and ethanolamine, respectively. Carboxylic acid-terminated second- and third-generation poly(amidoamine)-type heptaisobutylPOSS-core dendrons (that is, G2POSS-COOH and G3POSS-COOH, respectively) were quantitatively obtained by treatment of second- and third-generation $t$-butyl ester-POSS-core dendrons with $\mathrm{HCO}_{2} \mathrm{H}$. The hydrodynamic sizes of the micelles formed by the POSS-core dendrons (that is, G2POSS-OH and G3POSS-OH) in methanol decreased from $18 \pm 10$ to $6 \pm 4 \mathrm{~nm}$, respectively. Casting of a methanol solution of G3POSS-OH on a glass substrates followed by heating at $100^{\circ} \mathrm{C}$ for $15 \mathrm{~min}$ provided optically transparent films. However, casting of a chloroform solution resulted in an opaque whitish film. G2POSS-OH formed opaque whitish films from both methanol and chloroform solutions. Casting of a $2.6 \mathrm{M}$ formic acid solution of G3POSS-COOH and subsequent heating at $100{ }^{\circ} \mathrm{C}$ for $15 \mathrm{~min}$ generated a free-standing film with high transparency. The peripheral functional groups may have been exposed to the surface of the cast films; thus, the wettability of the films was controlled via the polarity of the $\mathrm{COOH}$ groups.

\section{ACKNOWLEDGEMENTS}

This study is part of a Grant-in-Aid for Scientific Research (No. 24350058) from the Ministry of Education, Culture, Sports, Science and Technology, Government of Japan, and a Grant-in-Aid for Scientific Research on Innovative Areas ('New Polymeric Materials Based on Element-Blocks (No.2401)') (24102003) of The Ministry of Education, Culture, Sports, Science and Technology, Japan. We thank Professor Tsuyoshi Kawai of Nara Institute of Science and Technology for MALDI-TOF-MS measurements supported by the Nanotechnology Platform. We also thank Professor Q Tran-Cong-Miyata of Kyoto Institute of Technology for measurement contact angle measurements.

1 Cordes, D. B., Lickiss, P. D. \& Rataboul, F. Recent developments in the chemistry of cubic polyhedral oligosilsesqioxanes. Chem. Rev. 110, 2081-2173 (2010).

2 Laine, R. M. Nanobuilding blocks based on the $\left[\mathrm{OSiO}_{1.5}\right]_{(\mathrm{x})}(\mathrm{x}=6,8,10)$ octasilsesquioxanes. J. Mater. Chem. 15, 3725-3744 (2005).

3 Tanaka, K. \& Chujo, Y. Advanced functional materials based on polyhedral oligomeric silsesquioxane (POSS). J. Mater. Chem. 22, 1733-1746 (2012).

$4 \mathrm{Kim}, \mathrm{K}$. M. \& Chujo, Y. Liquid-crystalline organic-inorganic hybrid polymers with functionalized silsesquioxanes. J. Polym. Sci. A Polym. Chem. 39, 4035-4043 (2001).

5 Mitsuishi, M., Zhao, F., Kim, Y., Watanabe, A. \& Miyashita, T. Preparation of ultrathin silsesquioxane nanofilms via polymer Langmuir-Blodgett films. Chem. Mater. 20, 4310-4316 (2008).

6 Wahab, M. A., Mya, K. Y. \& He, C. O. Synthesis, morphology, and properties of hydroxyl terminated-POSS/polyimide low-k nanocomposites films. J. Polym. Sci. Polym. Chem. 46, 5887-5896 (2008)
7 Tanaka, K., Adachi, S. \& Chujo, Y. Structure-property relationship of octa-substituted POSS in thermal and mechanical reinforcements of conventional polymers. J. Polym. Sci. A Polym. Chem. 47, 5690-5697 (2009).

8 Choi, J., Yee, A. F. \& Laine, R. M. Toughening of cubic silsesquioxane epoxy nanocomposites using core-shell rubber particles: a three-component hybrid system. Macromolecules 37, 3267-3276 (2004).

9 Zhang, C., Babonneau, F., Bonhomme, C., Laine, R. M., Soles, C. L., Hristov, H. A. \& Yee, A. L. Highly porous polyhedral silsesquioxane polymers. Synthesis and characterization. J. Am. Chem. Soc. 120, 8380-8391 (1998).

10 Lin, H., Qu, J., Zhang, Z., Dong, J. \& Zou, H. Ring-opening polymerization reaction of polyhedral oligomeric silsesquioxanes (POSSs) for preparation of well-controlled 3D skeletal hybrid monoliths. Chem. Commun. 49, 231-233 (2013).

11 Lichtenhan, J. D., Otonari, Y. A. \& Cam, M. J. Linear hybrid polymer building-blocksmethacrylate-functionalized polyhedral oligomeric silsesquioxane monomers and polymers. Macromolecules 28, 8435-8437 (1995).

12 Zheng, L., Hong, S., Cardoen, G., Burgaz, E., Gido, S. P. \& Coughlin, E. B. Polymer nanocomposites through controlled self-assembly of cubic silsesquioxane scaffolds. Macromolecules 37, 8606-8611 (2004)

13 Ahn, B., Hirai, T., Jin, S., Rho, Y., Kim, K.-W., Kakimoto, M., Gopalan, P., Hayakawa, T. \& Ree, M. Hierarchical structure in nanoscale thin films of a poly (styrene- $b$-methacrylate grafted with POSS) (PS $214-b-P M A P O S S_{27}$ ). Macromolecules 43, 10568-10581 (2010).

$14 \mathrm{Wu}$, J., Ge, Q. \& Mather, P. T. PEG-POSS multiblock polyurethanes: synthesis characterization, and hydrogel formation. Macromolecules 43, 7637-7649 (2010).

15 Lee, J., Cho, H.-J., Jung, B.-J., Cho, N. S. \& Shim, H.-K. Stabilized blue luminescent polyfluorenes: introducing polyhedral oligomeric silsesquioxane. Macromolecules 37, 8523-8529 (2004).

16 Pyun, J. \& Matyjaszewski, K. The synthesis of hybrid polymers using atom transfer radical polymerization: homopolymers and block copolymers from polyhedral oligomeric silsesquioxane monomers. Macromolecules 33, 217-220 (2000).

17 Escudé, N.C. \& Chen, E.Y.-X. Stereoregular methacrylate-POSS hybrid polymers: synthesis and nanostructured assemblies. Chem. Mater. 21, 5743-5753 (2009).

$18 \mathrm{Kim}$, K.-M., Keum, D.-K. \& Chujo, Y. Organic-inorganic polymer hybrids using polyoxazoline intiated by functionalized silsesquioxane. Macromolecules 36 867-875 (2003).

19 Tan, B. H., Hussain, H. \& He, C. B. Tailoring micelle formation and gelation in (PEG-P (MA-POSS) amphiphilic hybrid block copolymers. Macromolecules 44, 622-631 (2011).

20 Zhang, W., Fang, B., Walther, A. \& Müller, A. H. E. Synthesis via RAFT polymerization of tadpole-shaped organic/inorganic hybrid poly(acrylic acid) containing polyhedra oligomeric silsesquioxane (POSS) and their self-assembly in water. Macromolecules 42 , 2563-2569 (2009).

21 Zhang, W., Yuan, J., Weiss, S., Ye, X., Li, C. \& Müller, A. H. E. Telechelic hybrid poly (acrylic acid)s containing polyhedral oligomeric silsesquioxane (POSS) and their selfassembly in water. Macromolecules 44, 6891-6898 (2011).

22 Hussain, H., Tan, B. H., Seah, G. L., Liu, Y., He, C. B. \& Davis, T. P. Micelle formation and gelation of (PEG P(MA-POSS) amphiphilic block copolymers via associative hydrophobic effects. Langmuir 26, 11763-11773 (2010).

23 Jiang, B., Tao, W., Lu, X., Liu, Y., Jin, H., Pang, Y., Sun, X., Yan, D. \& Zhou, Y. A POSS based supramolecular amphiphile and its hierarchical self-assembly behaviors. Macromol. Rapid Commun. 33, 767-772 (2012).

24 Yu, X., Zhong, S., Li, X., Tu, Y., Yang, S., Van Hom, R. M., Ni, C., Pochan, D. J., Quirk, R. P., Wesdemiotis, C., Zhang, W.-B. \& Cheng, S. Z. D. A giant surfactant of polystyrene-(carboxylic acid-functionalized polyhedral oligomeric silsesquioxane) amphiphile with highly stretched polystyrene tails in micellar assemblies. J. Am. Chem. Soc. 132, 1674-16744 (2010).

25 Zheng, L., Waddon, A. J., Farrris, R. J. \& Coughlin, E. B. X-ray characterizations of polyethylene polyhedral oligomeric silsesquioxane copolymers. Macromolecules 35 , 2375-2379 (2002).

26 Zheng, L., Hong, S., Cardoen, G., Burgaz, E., Gido, S. P. \& Coughlinm, E. B. Polymer nanocomposites through controlled self-assembly of cubic silsesquioxane scaffolds. Macromolecules 37, 8606-8611 (2004).

27 Park, C., Lee, J. \& Kim, C. Functional supramolecular assemblies derived from dendritic building blocks. Chem. Commun. 47, 12042-12056 (2011).

28 Rosen, B. M., Wilson, C. J., Wilson, D. A., Peterca, M., Imam, M. R. \& Percec, V. Dendron-mediated self-assembly, disassembly, and self-organization of complex systems. Chem. Rev. 109, 6275-6540 (2009).

29 Kim, M.-K., Jeon, Y. M., Jeon, W. S., Kim, H.-J., Hong, S. G., Park, C. G. \& Kim, K. Novel dendron-stabilized gold nanoparticles with high stability and narrow size distribution. Chem. Commun. 37, 667-668 (2001).

30 Wang, R., Yang, J., Zheng, Z., Carducci, M. D., Jiao, J. \& Seraphin, S. Dendron-controlled nucleation and growth of gold nanoparticles. Angew. Chem. Int. Ed. 40, 549-552 (2001).

31 Chang, G.-P., Jeng, R.-J., Dai, S. A. \& Liu, Y.-L. Preparation and supramolecular selfassembly of amphiphilic Dendron-POSS nanohybrids. J. Nanosci. Nanotech. 9 4623-4632 (2009).

32 Naka, K., Fujita, M., Tanaka, K. \& Chujo, Y. Water-soluble anionic POSS-core dendrimer: synthesis and copper(II) complexes in aqueous solution. Langmuir 23 9057-9063 (2007)

33 Waddon, A. J. \& Coughlin, E. B. Crystal structure of polyhedral oligomeric silsesquioxane (POSS) nano-materials: a study by $x$-ray diffraction and electron microscopy. Chem. Mater. 15, 4555-4561 (2003).

Supplementary Information accompanies the paper on Polymer Journal website (http://www.nature.com/pj) 